\title{
LA RHÉTORIQUE RÉVOLUTIONNAIRE À L'ÉPREUVE DES MINORITÉS SEXUELLES DANS QUATRE PAMPHLETS DE LA RÉVOLUTION FRANÇAISE
}

La notion même de minorité sexuelle qui apparaît dans mon titre est un concept problématique. En effet, si on analyse les résultats du fameux rapport Kinsey ${ }^{1}$, pour ne prendre que cet exemple, la diversité des comportements sexuels se laisse difficilement circonscrire par des binarismes tels que majorité $v s$ minorité. Il semble plus juste de décrire la sexualité humaine comme un continuum et non pas en termes dualistes. J'adopte donc le concept de $\ll \mathrm{mi}-$ norité sexuelle » surtout pour des raisons pragmatiques car il permet d'englober l'ensemble des non-conformistes sexuels c'est-à-dire tous ceux et celles qui ne s'inscrivent pas dans le « cercle vertueux » de la sexualité « hétérosexuelle, conjugale, monogame, procréatrice et non commerciale », selon l'expression de Gayle S. Rubin ${ }^{2}$.Je me pencherai sur la façon dont la rhétorique de la libération et des droits de l'homme, caractéristique de la Révolution française, s'accommode des pratiques érotiques minoritaires, surtout des relations non procréatrices et des rapports sexuels commerciaux, en examinant quatre pamphlets révolutionnaires où il en est question, parus dans les années 1790-1791 et présentant une certaine unité, notamment thématique ${ }^{3}$. Il s'agira des textes : Les Enfans ${ }^{4}$ de Sodome à l'Assemblée Nationale, Requête et décret en faveur des putains, des fouteuses,

1 Voir p. ex. D. Eribon (éd.), Dictionnaire des cultures gays et lesbiennes, Paris, Larousse, 2003, p. 275-276.

2 C'est ainsi que la définit en raccourci Rubin dans son texte désormais classique « Penser le sexe. Pour une théorie radicale de la politique de la sexualité », [in] J. Butler, G. S. Rubin, Marché au sexe, trad. E. Sokol et F. Bolter, Paris, EPEL, 2002, p. 86.

3 À ce propos, voir P. Cardon, « Présentation », [in] Les Enfans de Sodome à l'Assemblée Nationale, Lille, Les Cahiers QuestiondeGenre/GKC, 2005, p. 11-12. Les citations suivantes de cette édition, qui comprend les quatre pamphlets analysés, seront localisées par le numéro de la page, donné entre parenthèses dans le corps du texte.

4 Je respecte ici, comme dans les citations qui suivent, l'orthographe originale, y compris en ce qui concerne l'usage des majuscules. 
des macquerelles et des branleuses contre les bougres, les bardaches et les brûleurs de paillasses, Les Petits Bougres au Manège et La Liberté ou $M^{\text {lle }}$ Raucourt. Comment ces pamphlets parlent-ils des minorités sexuelles ? Dans quelle mesure innovent-ils dans le traitement de ce sujet ? Les nouvelles valeurs de liberté, d'égalité et de fraternité s'appliquent-elles à la sexualité ? Qu'est-ce que la Révolution fait aux minorités sexuelles et qu'est-ce que ces dernières font à la Révolution ?

Premièrement, les pamphlets participent de la culture politique de la Révolution. Les bouleversements révolutionnaires stimulent une culture basée sur le débat et la polémique que ces pamphlets reproduisent à leur manière. Le fait même qu'ils se répondent ${ }^{5}$ et forment une sorte de suite logique en fait des écrits typiques de cette période. Les Petits Bougres... se présentent ainsi explicitement telle une réponse à la Requête..., La Liberté... fait directement et indirectement référence aux trois autres textes. Le pamphlet est l'un des genres caractéristiques de cette culture politique ${ }^{6}$ révolutionnaire dans laquelle il s'agit de faire triompher son point de vue et de discréditer ses adversaires. C'est probablement sous l'influence de cette ambiance de polémique généralisée, générée par la vie politique révolutionnaire, que les pamphlétaires présentent une vision conflictuelle des rapports entre différentes minorités sexuelles ; p. ex. les prostituées demandent des mesures contre les « bougres », comme le port de marques vestimentaires susceptibles de les faire identifier, ce à quoi ces derniers répondent en réclamant notamment d'imposer une visite médicale aux catins et d'enfermer celles qui sont malades à Bicêtre. De ces échanges polémiques par moments très violents et dont la portée dépasse la seule question des intérêts des minorités sexuelles ${ }^{7}$ ne se dégage donc pas quelque conception idéaliste de la démocratie nouvellement instaurée ; celle-ci est plutôt envisagée comme un jeu assez brutal de forces antagonistes qui essaient d'influencer le législateur chargé de fixer de nouvelles règles de vie en société.

Les auteur(e)s anonymes donnent donc la parole à des représentant(e)s des minorités sexuelles, parfois personnages historiques, comme $\mathrm{M}^{\mathrm{Ile}}$ Raucourt, ac-

5 À ce propos, voir aussi les remarques de P. Cardon, op. cit., p. 12-13.

${ }^{6}$ En témoignent p.ex. les entrées qui lui sont consacrés dans le Dictionnaire des genres et notions littéraires, Paris, Encyclopaedia Universalis/Albin Michel, 2001, p. 549550, et J. Sławiński (éd.), Słownik terminów literackich, Wrocław, Zakład Narodowy im. Ossolińskich, 2008, p. 368-369.

${ }^{7}$ Les auteur(e)s ne se contentent pas de faire prêcher aux minorités sexuelles leurs propres causes, ils les font aussi participer à des débats politiques plus larges. Ainsi le discours de $\mathrm{M}^{\text {lle }}$ Raucourt, dans le pamphlet où elle apparaît, commence-t-il d'une part par l'adhésion aux valeurs de liberté et d'autre part par une dénonciation assez longue de la violence révolutionnaire. En lui répondant, la vicomtesse de Con-fendu, représentante des prostituées, l'accuse de connivence avec l'aristocratie et, par contraste, présente les prostituées comme des « patriotes zélées $»($ p. 121). 
trice célèbre, pour qu' ils argumentent en faveur de la légitimité de leurs comportements érotiques, prohibés sous l'Ancien Régime. On connaît les liens entre la philosophie rationaliste des Lumières et l'idéologie révolutionnaire, or les pamphlétaires prêtent justement à leurs personnages des arguments puisés dans le discours philosophique de l'époque, surtout dans la philosophie de la Nature. Selon l'affirmation d'Albert Soboul, la Révolution « se plaça [...] sous l'invocation du droit naturel $\gg^{8}$. Les personnages font de même pour légitimer leurs désirs et pratiques sexuelles. Ils recourent à toute une phraséologie héritée des philosophes. Dans son discours, qui fait partie des Enfans de Sodome..., l'un d'eux, le duc de Noailles, oppose ainsi « l'ignorance des siècles » aux « lumières de la philosophie », appelle à « anéantir jusqu'aux moindres vestiges des préjugés » (p. 50) et présente la législation contre la sodomie comme des « lois criminelles » (p. 51). Prétendant rompre avec l'ancien discours du « contre-nature $»$, et soutenant que « le Concubinage n' [est] pas plus naturel que l'anti-physique » (p. 52), il emploie pourtant un terme qui, étymologiquement, en est l'exact équivalent ; il fait toutefois plus scientifique, ayant été utilisé par les philosophes ${ }^{9}$. Dans le même pamphlet, à la rationalisation de Dieu, transformé en Être Suprême, est associée la dédramatisation de la sodomie, présentée comme une «bagatelle » (p. 41) dont la divinité ne s'occupe plus. Dans l'« Intervention des tribades dans la cause des bougres \& des bardaches contre les fouteuses \& C. », qui fait partie de La Liberté..., les rapports entre femmes sont également rationalisés dans l'esprit des Lumières par leur présentation comme moyen d'éviter les grossesses indésirables. Dans son discours, $\mathrm{M}^{\mathrm{lle}}$ Raucourt les légitime aussi en recourant à des arguments historiques (elle invoque l'exemple des Amazones, des dames romaines et des femmes sauvages du Canada). Quant à la prostitution, dans sa réponse à $\mathrm{M}^{\mathrm{lle}} \mathrm{Raucourt}$, la vicomtesse de Con-fendu la justifie également par une argumentation à la fois naturaliste et économique.

Mais les pamphlétaires attribuent surtout à leurs personnages la volonté, typiquement révolutionnaire, de trouver une traduction politique, et plus précisément législative, à la critique philosophique des préjugés concernant les sexualités minoritaires qu'ils effectuent. Ils imaginent les non-conformistes sexuels comme des citoyens très actifs en leur prêtant la prétention d'inscrire la liberté sexuelle dans la loi. Dans Les Enfans de Sodome..., d'un discours rationaliste et progressiste découlent des revendications rédigées sous forme d'articles selon lesquels « il sera permis à tout Chevalier de la Manchette d'user de sa personne » (p. 53).

${ }^{8}$ A. Soboul, « Réalités et idées neuves », [in] P. Barbéris, C. Duchet (coord.), Manuel d'histoire littéraire de la France. Tome IV, 1789-1848, premier volume, Paris, Éd. Sociales, 1972, p. 17-58, p. 19 pour la citation.

9 Sur l'étymologie du mot « antiphysique » et ses usages au XVIII ${ }^{e}$ siècle, voir C. Courouve, Vocabulaire de l'homosexualité masculine, Paris, Fayard, p. 56-58. 
La liberté sexuelle, considérée comme corollaire de la liberté individuelle, est ainsi inscrite dans un texte officiel que ses auteurs proposent de soumettre à l'Assemblée afin qu'elle le valide. Au-delà des déclarations de principe, les « enfants de Sodome » et les prostituées demandent aussi à l'Assemblée des dispositions législatives concrètes. Le prétendu décret pris par les députés en faveur des catins est d'ailleurs reproduit à la suite de leur requête. La sexualité se trouve ainsi politisée.

Cette politisation s'effectue non seulement à travers la revendication d'inscrire la liberté sexuelle dans la loi, mais aussi par le recours à un répertoire de situations et de formes discursives typiques de la vie politique et de l'activité législative à la Révolution. Ce qui revient le plus souvent, c'est une scénographie parlementaire. Les Enfans de Sodome... présentent même le contexte historique de l'essor du parlementarisme : à la suite de la convocation des États Généraux, toutes sortes d'assemblées ont foisonné. L'auteur(e) mentionne parmi elles celle de l'Ordre de la Manchette, composée de « Bougres, Bardaches, Bardachins, Bardachinets et Tribades $\gg($ p. 46), termes qui renvoient aux relations de même sexe. Il donne un compte rendu de leurs débats où sont notamment transcrits les discours de plusieurs membres. À titre d'exemple, Tabouret, une tribade, prononce un discours où elle entend prouver son droit à siéger dans cette assemblée. Les autres activités des personnages sont aussi typiquement celles des membres d'une assemblée politique : on fixe la façon de voter et on nomme deux présidents dont chacun prononce à son tour un discours. Après quoi, l'assemblée décrète les sept articles dans lesquels elle décrit les droits des « Chevaliers de la Manchette ». Elle élit aussi ses députés à l'Assemblée Nationale. Les personnages exercent différentes fonctions publiques : président, vice-président, secrétaire, député, membre du Comité Civil, du Comité Militaire, du Comité des Commissaires Enquêteurs et Examinateurs, du Comité Ecclésiastique ou du Comité des Rapports. Placés dans des situations officielles, ils recourent à des modes de raisonnement et de discours adaptés aux circonstances. Par exemple, Les Petits Bougres... contiennent une réponse des « bougres » à la Requête..., avec une argumentation juridique destinée à contrer les prétentions des prostituées, et prouver l'illégalité du décret de l'Assemblée contre les « enfants de Sodome $\gg$. Les personnages accomplissent aussi des actes de procédure. La réponse des « bougres » est signifiée aux auteures de la requête par exploit d'huissier. Une procuration en bonne et due forme, donnée par les membres de la « Société Sodomique $\gg$ au marquis de Villette, pour agir en son nom, fait aussi partie du texte des Petits Bougres... ; elle est accompagnée de sa légalisation. À l'intervention écrite des tribades dans l'affaire des « bougres » et « bardaches » contre les prostituées est également joint l'exploit de sa signification à ces dernières par voie d'huissier. Pour résumer, les personnages des pamphlets sont placés dans des situations caractéristiques de la nouvelle époque, telles qu'une assemblée politique, ses débats, le vote, la désignation de ses représentants pour l'Assemblée 
Nationale ou la formulation de demandes qui lui seront adressées. Ils pratiquent des formes discursives qui y restent associées : le discours politique, la requête, la motion, le décret, en recourant à toute une phraséologie, notamment juridique, qui leur est propre. Ils illustrent ainsi cette formule contenue dans l'article VII du décret que les « enfants de Sodome » soumettent à l'Assemblée Nationale : $\ll[\ldots]$ on peut être Bougre et Citoyen et $[\ldots]$ les affaires de Cul n'empêchent et ne peuvent empêcher de se montrer ardent pour les affaires de la Patrie $\gg($ p. 55).

Comme le suggère cette dernière formule, les situations, les institutions, la rhétorique et les idéaux révolutionnaires ainsi que le langage juridique sont toutefois tournés en dérision dans ces textes, surtout par leur sexualisation ou, plus exactement, pornographisation ${ }^{10}$. La sexualité, traditionnellement associée sous l'Ancien Régime au registre bas, est ici en plus décrite d'une manière particulièrement vulgaire. Un contraste apparaît entre celle-ci d'une part et la grandiloquence révolutionnaire ainsi que la phraséologie juridique d'autre part. $\mathrm{Du}$ heurt des registres bas et élevé résulte une impression de parodie. Le titre de l'un des pamphlets peut exemplifier ce heurt dans la mesure où à des formes de discours officielles « requête et décret » s'y trouvent accolés des termes obscènes renvoyant au monde de la prostitution : «putains », « fouteuses 》, « macquerelles $\gg($ sic $)$ et « branleuses ». Sur la page de titre du même ouvrage, il est indiqué qu'il « se trouve chez toutes les Fouteuses Nationales » (p. 77). Comme on le sait, « la Révolution [...] a [...] renouvelé le vocabulaire $»^{11}$; ici, l'un des termes-phares de la rhétorique révolutionnaire, la «nation », apparaissant sous forme adjectivale, est ridiculisé par un emploi pornographique. Des formes officielles ou des valeurs nobles, comme les droits de l'homme (dont il est question p.ex. p. 53), se trouvent ainsi régulièrement compromises par leur insertion dans des contextes obscènes. Tous les pamphlets foisonnent de termes anatomiques ou de leurs équivalents populaires ou graveleux, tels « anus » (p. 44), « cul » (p. 48), « con » (p. 72), « couilles » (p. 79), « vit » (p. 79), « fesses » (p. 81), «tétons » (p. 82), « clitoris » (p. 109), « pine » (p. 111); l'acte sexuel est décrit à l'aide de verbes et expressions obscènes : « sodomiser » (p. 52), « bougrifier » (p. 52), « baiser » (p. 64), « foutre » (p. 71), « gamahucher en cul et en con » (p. 72), « se branler » (p. 81), « décharger » (p. 83), « enculer » (p. 92), «éjaculer $\gg($ p. 112), « foutrailler » (p. 122) ; le mot savant « anti-physique » est concurrencé par ses synonymes plus traditionnels et moins nobles, comme « sodomie » (p. 44) ou « bougrerie » (p. 41); les amateurs de ces plaisirs sont des «Anti-physiciens » (p. 51), « Bougres, Bardaches, Bardachins, Bardachinets »

10 Il convient de rappeler que le terme « pornographe » date du XVIII ${ }^{\mathrm{e}}$ siècle et qu'il a été forgé par Restif de La Bretonne. À ce sujet, voir p. ex. D. Maingueneau, La Littérature pornographique, Paris, Armand Colin, 2007, p. 9.

11 A. Soboul, op. cit., p. 34. 
(p. 46) ou encore « enculeurs » (p. 91), « sodomistes » (p. 92), « rivettes » (p. 113), leurs amatrices - «Tribades » (p. 46) et « anandrines » (p. 120); les prostituées - « putains $\gg, ~<$ fouteuses $\gg, ~ «$ macquerelles $\gg, ~<$ branleuses $\gg$, « garces $»$, « gouines $\gg($ p. 96).

La politique elle-même est sexualisée : les adeptes de l'《 anti-physique » forment un « parti $\gg$, alors que ceux et celles qui n'en sont pas, se trouvent dans le « parti de l'opposition » (p. 54). Dans la Requête..., les députés sont désignés par l'expression obscène « nos fouteurs de l'assemblée nationale » (p. 82) et présentés comme des clients des prostituées. L'auteur(e) imagine aussi que ladite assemblée comporte un «Comité de Fouterie » et que le grand sceau de la Nation porte « l'empreinte d'un vit majestueux, barbouillant voluptueusement de foutre les lèvres vermeilles d'un con couronné d'une guirlande de couilles » (p. 82). Les anandrines ont aussi leur sceau « portant l'empreinte d'un clitoris imperceptible au milieu d'un large con $\gg$ (p. 118). Dans Les Petits Bougres..., apparaissent des institutions officielles fantaisistes telles que la « Société Sodomique », le «Conseil Général des Bougres et des Bardaches » ou « le greffe foutatif » et les personnages endossent des charges comme « huissier de la Manchette $\gg$ ou « notaire enculeur $\gg$. Même les fonctions et les emblèmes officiels revêtent donc un caractère sexuel. Dans la Requête..., le calendrier révolutionnaire lui-même est parodié car l'ère nouvelle qu'il est censé signaler est sexualisée à travers la date d'édition donnée : «L'an second de la régénération foutative ». Dans Les Enfans de Sodome..., les débats tournent à l'orgie et le vote se fait par « coups de cul ». Dans Les Petits Bougres..., les délibérations du Conseil Général des Bougres et des Bardaches et la rédaction de la procuration se transforment aussi en partie fine. Dans La Liberté..., les anandrines votent en faisant l'amour. Même certains actes de procédure sont pornographisés, p.ex. quand le compte rendu des délibérations du Conseil Général des Bougres et des Bardaches se termine par la formule suivante : «ceux qui n’ont su signer, ayant déchargé sur le registre $\gg$ (p. 101) ; ou encore, lorsqu'un certain L. M. indique à la suite de la formule de légalisation avoir reçu, en plus de la somme requise, « une ondée de foutre dans les fesses $\gg$ (p. 103) ; et enfin, quand les anandrines signent « avec [leur] foutre $\gg$ (p. 118). Les institutions, fonctions, formes et actes officiels sont tous annexés par la sexualité dans le cadre d'un pansexualisme pornographique.

En tournant en dérision la vie politique et la rhétorique révolutionnaire par leur pornographisation, les pamphlets prolongent une tradition discursive de l'Ancien Régime. En effet, il existait alors une pratique de la polémique politique et religieuse dans laquelle on discréditait son adversaire en lui prêtant des goûts sexuels peu orthodoxes ${ }^{12}$. Avec Lech Nijakowski, on pourrait parler à ce propos

12 À ce sujet, voir p. ex. G. Poirier, L'Homosexualité dans l'imaginaire de la Renaissance, Paris, H. Champion, 1996. 
d'une $\ll$ critique pornographique $\gg^{13}$ de l'ordre politique existant, en y ajoutant que de cet usage de la sexualité découlait une certaine solidarité entre le genre du pamphlet et la thématique des minorités sexuelles. Le constat que des auteur(e) $s$ de l'époque révolutionnaire recourent à ce genre traditionnel n'explique toutefois pas quelle était la cible de leurs attaques et leur intention. Les chercheurs qui se sont jusqu’à présent penchés sur ces textes ont apporté des réponses contradictoires à la question de savoir qui était visé dans les pamphlets que j'analyse $\mathrm{ici}^{14}$. À première vue, dans la mesure où toute la rhétorique révolutionnaire y est ridiculisée, on pourrait supposer que les pamphlétaires poursuivent une visée contre-révolutionnaire. Toutefois, sur les listes des sodomites et des tribades qui font partie des Enfans de Sodome..., toutes les classes sociales, y compris les anciennes élites, sont présentes. Si on y ajoute le fait que toutes les minorités et leurs pratiques y soient désignées par des termes obscènes ou dégradants, on peut émettre l'hypothèse que les auteur(e)s anonymes étaient des contre-révolutionnaires moins en termes strictement politiques que sexuels. J'ai déjà signalé que les personnages des pamphlets faisaient appel à toute une rhétorique héritée des Lumières. Il faut y ajouter que, dans la réalité extratextuelle, cette rhétorique a porté ses fruits dans la mesure où la période révolutionnaire peut être vue comme le couronnement de cet effort de rationalisation de l'approche des sexualités minoritaires. En 1791, l'Assemblée constituante dépénalise le crime de sodomie, renvoyant à toute forme de relation non procréatrice ${ }^{15}$. Comme l'ont montré les recherches de Clyde Plumauzille, la Révolution a aussi dépénalisé la prostitution par « l'imposition d'un régime juridique libéral $»^{16}$. Même si les pratiques policières vont parfois à l'encontre de la nouvelle législation, celle-ci évolue indéniablement. Il me semble que les auteur(e)s des pamphlets analysés avaient pour objectif de limiter cette évolution.

Les pamphlets contiennent aussi d'autres résidus discursifs de l'Ancien Régime. Il s'agit notamment de toute une rhétorique religieuse traditionnellement appliquée aux sexualités minoritaires, et surtout aux relations de même sexe. La présence de cette matrice discursive religieuse témoigne de la prégnance de la religion dans les mentalités de l'époque, malgré tout l'aspect anticlérical de la Révo-

13 Voir son livre, op. cit., surtout p. 119 et suiv.

14 T. Pastorello (voir son article « La sodomie masculine dans les pamphlets révolutionnaires $\gg, A H R F, \mathrm{n}^{\circ} 361$, p. 91-130) prétend que c'étaient les anciennes élites (clergé et aristocratie), alors que P. Cardon présente Les Enfans.. . « comme des attaques à caractère homophobe contre les Jacobins $\gg$ (voir P. Cardon, op. cit., p. 13).

15 Sur la dépénalisation, voir F. Leroy-Forgeot, Histoire juridique de l'homosexualité en Europe, Paris, PUF, 1997, surtout p. 64 et suiv.

16 Voir le compte rendu de sa thèse, www.genrehistoire.revues.org (page consultée le 28 janvier 2016). 
lution. À côté de la veine parodique susmentionnée, le discours religieux (théologique et moral) était un autre locus discursif associé sous l'Ancien Régime aux sexualités minoritaires. La rhétorique religieuse que les pamphlets reconduisent comprend entre autres des figures empruntées à la Bible, p. ex. « Sodome » et ses dérivés (《 sodomie », « sodomiste $\gg, ~ 《$ sodomique $», ~ «$ sodomiser $\gg)$, «Gomorrhe » (p. 43), « apôtre » (p. 48 ; c'est l'abbé Viennet qui est qualifié d'«Apôtre de Sodome ») ou encore le Christ lui-même dont l'affection pour Saint Jean est interprétée dans un sens homosexuel (pp. 51-52); elle contient aussi tout un vocabulaire emprunté au langage religieux : « ordre $\gg($ p. 40), « novices $\gg($ p. 44), « prosélytes » (p. 46), « prêtresse » (p. 48 ; Tabouret est désignée par l'expression « prêtresse de Sodome $\gg)$, « martyr $\gg($ p. 51), « martyrologe $\gg($ p. 51), « culte $\gg($ p. 84), « secte $\gg($ p. 103), etc. Le modèle religieux reste donc de mise pour penser les sexualités minoritaires. Dans sa réponse aux anandrines, la vicomtesse de Con-fendu reprend même l'argument du contre-nature, popularisé par la théologie chrétienne $\mathrm{e}^{17}$, pour condamner les pratiques sexuelles entre femmes et, par contraste, légitimer celles des prostituées avec leurs clients. Comme, avec la Révolution, les mentalités n’ont bien évidemment pas changé du jour au lendemain, les pamphlets mélangent la nouvelle rhétorique avec des éléments de l'ancienne, d'origine essentiellement religieuse, bien que des figures mythologiques, comme Ganymède (p. 43), y apparaissent aussi. La rupture entre l'ancien et le nouveau régime est donc loin d'être absolue au niveau discursif. L'analyse de la rhétorique des pamphlets où sont présentes diverses minorités sexuelles confirme ainsi, à une échelle plus large, ce que dit Didier Godard quant à la continuité de la situation des homosexuels entre l'ancienne et la nouvelle France. Il reprend « la thèse de Tocqueville, selon laquelle la Révolution et l'Empire, par rapport à l'Ancien Régime, constituent plutôt un prolongement et un aboutissement qu'une rupture $\gg^{18}$. Godard pense ici surtout aux pratiques, mais cette thèse se vérifie aussi au niveau des discours.

Pourtant, il me semble qu'il serait erroné de s'en tenir au seul constat du caractère parodique et réactionnaire de ces textes. Je ne suis pas sûr qu'il faille aller aussi loin que leur éditeur contemporain, militant LGBTQI Patrick Cardon, qui considère qu' il est possible de les lire comme « un plaidoyer » (p. 14), mais force est de constater que, parallèlement à l'ancien modèle discursif, ils en contiennent un autre : celui de la défense des droits des minorités sexuelles. On sait que le mouvement LGBTQI a souvent mobilisé et continue à utiliser la rhétorique des droits de l'homme, héritée des Lumières et de la Révolution, et que c'est proba-

17 Voir L.-G. Tin (éd.), Dictionnaire de l'homophobie, Paris, PUF, 2003, surtout p. $108-109$ et 307.

18 D. Godard, L'Amour philosophique. L'Homosexualité masculine au siècle des Lumières, Béziers, H\&O Éditions, 2005, p. 234. 
blement grâce à cette rhétorique qu'il a remporté ses plus grandes victoires. Les pamphlets analysés contiennent ainsi à l'état d'ébauche la stratégie discursive de ce mouvement. Mais que nous dit la tonalité parodique de ces textes de la Révolution elle-même ? Â mon sens, elle témoigne surtout du caractère charnière de l'époque révolutionnaire. Les acteurs de la Révolution, ancrés dans l'ancien monde, ne sont pas prêts à aller jusqu'au bout de la logique de la libération et des droits de l'homme. Le sujet des sexualités minoritaires agit comme une sorte de révélateur marquant les limites du projet révolutionnaire. Malgré certaines avancées, la Révolution française ne fut pas une « révolution sexuelle »; elle ne le fut pas pour les femmes, comme on le sait grâce notamment aux recherches de Joan W. Scott ${ }^{19}$, qui a montré les impasses et les paradoxes de la rhétorique des droits de l'homme; elle ne le fut pas non plus pour les minorités sexuelles. Dans ce sens, la question des sexualités minoritaires met à l'épreuve la rhétorique révolutionnaire : elle contribue à poser des limites au projet de la Révolution, en circonscrivant la sphère de ce qui est sérieusement envisageable par la dérision de ce qui ne l'est pas. $\mathrm{Si}$, dans « Français, encore un effort si vous voulez être républicains $\gg$ dans La Philosophie dans le boudoir de Sade, une révolution sexuelle est envisagée tout à fait sérieusement, elle ne l'est pas dans les pamphlets analysés. Toutefois, la Révolution et ses sources philosophiques, les Lumières, ont fourni aux minorités sexuelles un réservoir discursif leur permettant de revendiquer leurs droits d'une manière plus ou moins efficace, selon les pays et les traditions locales. Mise à l'épreuve des minorités, la rhétorique de la libération et des droits de l'homme se trouvant à la base de la Révolution, s'avère apte à rendre compte de revendications d'ordre sexuel. Les pamphlets font sur un mode parodique ce que les acteurs de Mai ' 68 feront sur un mode sérieux : ils politisent la sexualité. Ils présentent le libre exercice de celle-ci comme l'un des droits de l'homme et de la femme. Il a fallu attendre longtemps avant que ce droit n'ait pu être revendiqué sérieusement. L'histoire des minorités sexuelles pourrait ainsi être celle de l'extinction progressive d'un rire dont les éclats retentissaient encore bien fort pendant la Révolution française.

\section{When the revolutionary rhetoric meets sexual minorities An analysis of four pamphlets of the French Revolution}

The paper discusses the way the rhetoric of human rights, characteristic of the French Revolution, deals with the subject of sexual minorities. The Revolution can be seen as a transition period in which the revolutionary rhetoric collides some ancient images of

19 Voir p. ex. son livre La Citoyenne paradoxale. Les Féministes françaises et les droits de l'homme, trad. M. Bourdé, C. Pratt, Paris, Albin Michel, 1998. 
sexual minorities. The limits of this rhetoric are tested in pamphlets where these minorities appear and that are also full of ancient images: Les Enfans de Sodome à l'Assemblée Nationale, Requête et décret en faveur des putains, des fouteuses, des macquerelles et des branleuses contre les bougres, les bardaches et les brûleurs de paillasses, Les Petits Bougres au Manège and La Liberté ou $M^{\text {He }}$ Raucourt.

Keywords: French Revolution - pamphlet - sexuality - sexual minority

Mots-clés : Révolution française - pamphlet - sexualité - minorité sexuelle 